\title{
Digital Video Watermarking Methods Using DCT and SVD
}

\author{
Meaad M. Salih ${ }^{1 *}$; Eman F. Ahmed ${ }^{2}$; Manar A. Al-Abaji ${ }^{3}$ \\ ${ }^{1,2,3}$ Department of Computer Sciences, College of Education for Pure Science, University of Mosul, Mosul, Iraq \\ Email: ${ }^{1 *}$ meaad_muhammad@yahoo.com, ${ }^{2}$ Emanrafee6067@gmail.com, ${ }^{3}$ manar198051@gmail.com
}

(Received January 12, 2019; Accepted May 16, 2019; Available online March 01, 2020)

DOI: 10.33899/edusj.2020.164378, ( $)$ 2020, College of Education for Pure Science, University of Mosul.

This is an open access article under the CC BY 4.0 license (http://creativecommons.org/licenses/by/4.0/).

\begin{abstract}
:
In this search, two methods were used to include the watermark in the video. The first method was based on DCT (Discrete Cosine Transform), the second method was based on an algorithm SVD (Singular Value Decomposition) for the purpose of converting video to frequency domain. The process of embedding the watermark in both methods was done after the original video was divided into a set of frames, and one frame was divided into a block of $8 \times 8$ and the DCT on each block when using the first method and the SVD algorithm when using the second method. And then include the Bit Binary for the watermark inside the center of the cluster. Random selection of video frames and rows of watermark images has been adopted in both ways. The performance of the two methods was assessed using the experimental tests PSNR, MSE and NC.The experimental results show that both methods have achieved a good understanding and high resistance against various attacks, adopted Matlab 2013a language.
\end{abstract}

Keyword: Digital Video, watermark.

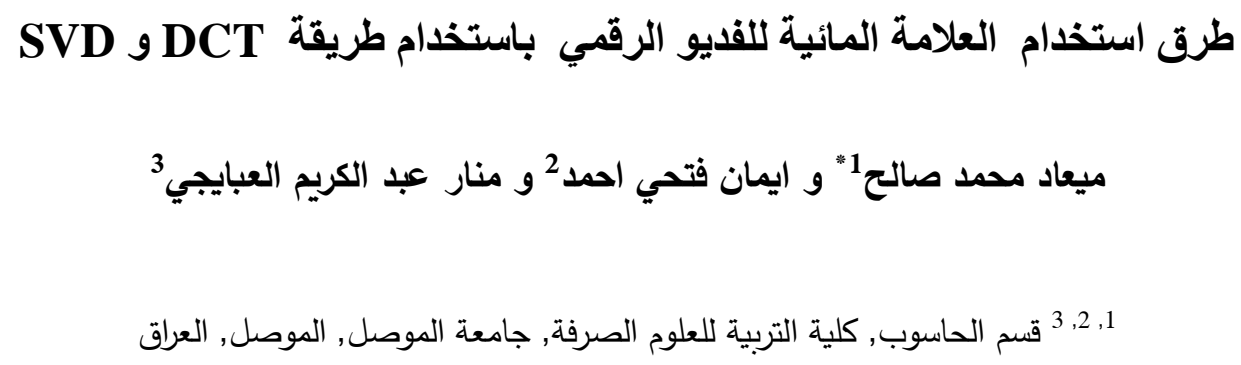

\footnotetext{
الملخص

في هذا البحث تم استخدام طريقتين لتضمين العلامة المائية في الفيديو. اعتمدت الطريقة الاولى على تحويل الجيب تمام SVD(Singular Value المتقطع (DCT(Discrete Cosine Transform) الطريقة الثانية اعتمدت على خوارزمية لغرض تحويل الفيديو الى المجال الترددي Fecomposition) الطريقتين تمت بعد تقسيم الفيديو الاصلي الى مجموعة من الاطارات Frames ثم تقسيم الاطار الواحد الى مجموعة من الكتل
} 


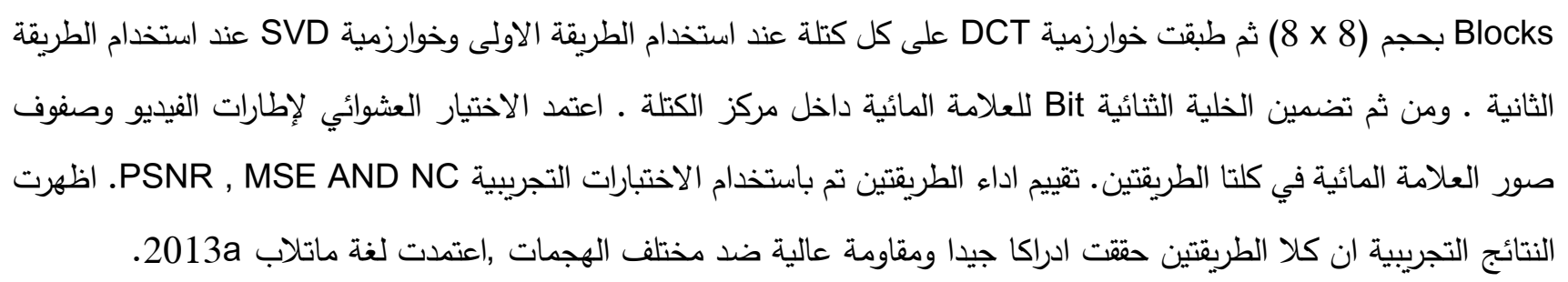

\section{Introduction}

Over the past years, there has been rapid progress in computers and in the Internet, where multimedia information such as images, video, text and audio has become available to network users and with a simple background, any information can be copied from the network. For the purpose of protecting individual property and copyright from theft, the watermark has been used for this purpose[1].

Digital watermark is a technique of embedding additional information in host data or messages are hidden within digital media such as video, images, text, and audio. Watermark is one of the interesting areas to develop a system with authentication and copyright protection methodology. Thus this technology can be used to protect copyright, piracy tracking, content[2] . The watermark is also used in security applications such as cards and secret communications such as the Ministry of Defense and Secret Intelligence[3].

Video watermarking methods can be divided into two groups spatial-domain and frequencydomain[4]. In spatial-domain is the process of embedding a watermark that is done directly by changing its pixel, while in frequency-domain the changing is done on the coefficients of video frames after transformed it according to the method of embedding. In this study, two non blind video watermarking methods were proposed; the first method used DCT And the second method used SVD [5].

Frequency Domain watermarking, most of watermarking methods the watermark will be inserted into frequency domain instead of the spatial domain for the robustness of the watermarking. Discrete Fourier Transformation DCT and Singular Value Decomposition SVD are the two main categories of data transformation in this domain[6].

\section{Related Studies}

Dhanashree S and Shedge [7] suggested Video watermarking that is designed and implemented as a secure and reliable scheme by comparing DWT-SVD and LWT-SVD.

Tahani Al-Khatib and Ali Al-Haj [8] suggested two mathematical transforms DWT and SVD. Both methods were combined to exploit their attractive features, Experimental results demonstrated the blindness and robustness of our proposed method as it successfully extracted the watermark from each frame without using the original video. 
Jitender Kumar AND Yogesh Rathi [9] proposed a new watermarking technique which involves embedding two or more messages or images in a single frame of video for the purpose of security and repeat the same process for $\mathrm{N}$-frames of the video for authentication of whole video, the results show that the PSNR of final output watermarked video is very good.

Chitrasen and Tanuja Kashyap [10] suggested, a Hybrid video watermark technique where DCT and 3rd level of DWT, is used. PSNR, MSE and NC values of Hybrid technique are analyzed on the basis of different noises and attacks. Hybrid technique is robust against high stream, low stream, frame drop, frame trim attacks The result shows that the proposed algorithm has a strong ability to resist different watermark attacks .

U.Shyamala Devi1,et.al. [11] suggested video watermarking is performed by using a hybrid DWT and SVD, The quality of the original frame and the embedded frame is estimated by means of MSE and PSNR.

\section{Singular Value Decomposition (SVD)}

SVD is a numerical method used to analyze matrices. In general if $A$ is a square matrix $n \times n$ then the SVD of A is defined by the formula:

$\mathrm{A}=\mathrm{US} \mathrm{V}$

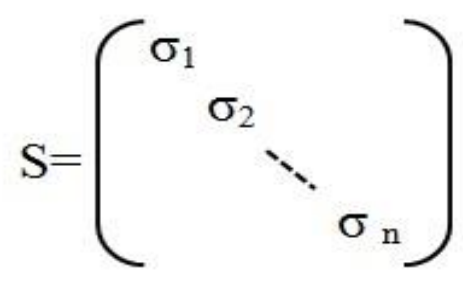

Where, $\mathrm{S} \in \mathrm{R}^{\mathrm{n} \times \mathrm{n}}$ represents a diagonal matrix, the diagonal elements in $\mathrm{S}$ are called the singular values of the matrix $A$ and are arranged in descending order $\sigma_{1} \geq \sigma_{2} \geq \sigma_{3} \geq \ldots . . \sigma_{\mathrm{r}} \geq \sigma_{\mathrm{r}+1} \geq \ldots \ldots \geq \sigma_{\mathrm{n}}$.

$\mathrm{U} \in \mathrm{R}^{\mathrm{n} \times \mathrm{n}}$ and $\mathrm{V} \in \mathrm{R}^{\mathrm{n} \times \mathrm{n}}$ are orthogonal matrix. The columns in $\mathrm{U}$ represent the left singular vectors, where as $\mathrm{V}$ columns represent the right singular vectors, both of them determine the geometry of the image while the diagonal elements in $\mathrm{S}$ represent luminance of image[8][3].

\section{Discrete Cosine Transform(DCT)}

DCT is considered one of many mathematical transfers that are used to unlink image elements ; DCT is considered the best method in the field of image energy hoarding in a few conversion coefficients. The conversion matrix is characterized by having the maximum value in the upper-left corner and the values of the coefficients are decrease towards right and down. the coefficients near the upper left corner of the conversion matrix represent low-frequency compounds (smoothed image), While the remaining coefficients represent high-frequency compounds (edges)[6].

The mathematical definition of two dimensional DCT performed on an $8 \times 8$ block is given in the following equation[11]. 
$F(u, v)$ is the DCT of $f(x, y)$.

$$
F(u, v)=\frac{C(u) C(v)}{4} \sum_{x=0}^{7} f(x, y) \cos \left\lceil\frac{\pi(2 x+1) u}{16}\right\rceil \cos \left\lceil\frac{\pi(2 y x+1) v}{16}\right\rceil
$$

where

$$
C(e)= \begin{cases}\frac{1}{\sqrt{2}} & \text { if } \mathrm{e}=0 \\ 1 & \text { if } \mathrm{e} \neq 0\end{cases}
$$

The mathematical definition of 2-dimensional inverse DCT performed on an $8 \times 8$ block of pixels is given in following equation [11].

$$
f(x, y)=\frac{1}{4} \sum_{u=0}^{7} \sum_{v=0}^{7} C(u) C(v) F(u, v) \cos \left[\frac{\pi(2 x+1) u}{16}\right] \cos \left[\frac{\pi(2 x+1) v}{16}\right]-----(2)
$$

\section{The Proposed Method:}

In this research Two methods are presented for digital video watermarking . The first is one based on DCT and the second one is based on SVD.

\subsection{DCT based on Watermark embedding algorithm}

Step1:Read watermark image.

Step2:Convert RGB watermark to GRAY.

Step3:Generate random numbers within the range 1-no. of rows in watermark.

Step4:Read video.

Step5:Generate random numbers within the range 1-no. of frames in video.

Step6:Convert the values of watermark rows to binary sequence (bin_seq).

Step7:Embedding binary sequence into random frames using the following steps:

is widely used in image and video coding because it exhibits high signal decorrelation rates and can be easily implemented on the majority of contemporary computing architectures . The key feature of the DCT $8 \times 8$ is that any pair of 8 X8 blocks can be processed independently . This make possible fully parallel implementation of DCT $8 \times 8$ by definition.

- Divide frame into blocks $8 \times 8$.

- Convert the blocks to DCT , and take the center of them.

- If (bin_seq(i) =1).

- then block center $=$ block center +1 .

- else no action. 
- end.

- apply Invers Discreet Cosine Transform (IDCT) to blocks.

Step9:rewrite the video .

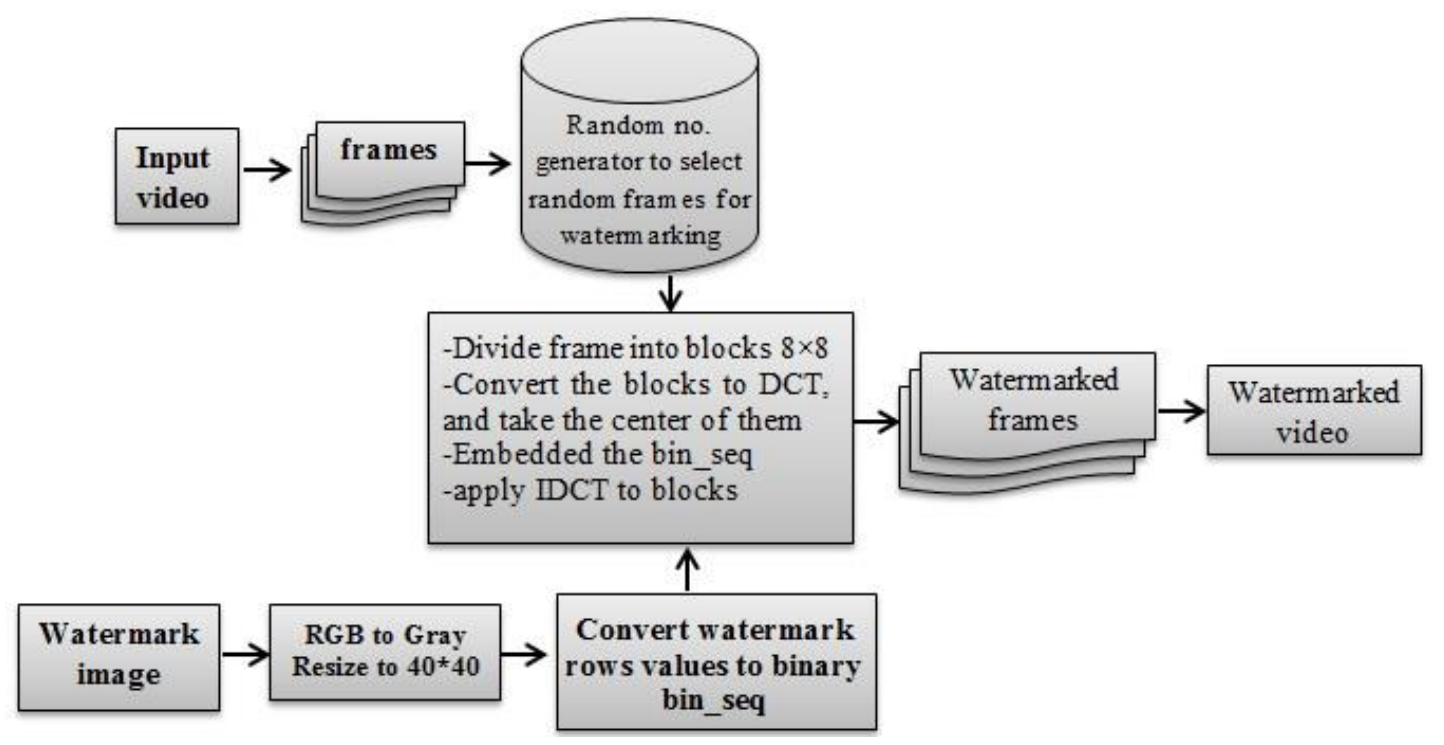

Figure(1) Watermark embedding algorithm based on DCT

\subsection{DCT based on Watermark Extraction algorithm}

Step1:Read the origin video(video1) and the watermarked video(video2).

Step2:Generate the same random numbers within the range 1-no. of frames depending on seek, and use them to take the frames from (video1) and (video2) .

Step3: Divide frames of video1\& video2 into blocks $8 \times 8$.

Step4:Convert the blocks of video1 to DCT (origin_block) \& the blocks of video2 to DCT (stego_block), calculate the difference (diff_block).

diff_block= origin block - stego_block.

Step5:Take the center of (diff_block) to create the extract text.

Text(i) $=$ round (diff_block $(4,4))$.

Step6:Convert the extracted bits to decimal values to create the rows of watermark.

Step7:Rearrange the rows to build the original watermark based on the 40 random numbers generated within the range 1-no. of rows using seeking. 


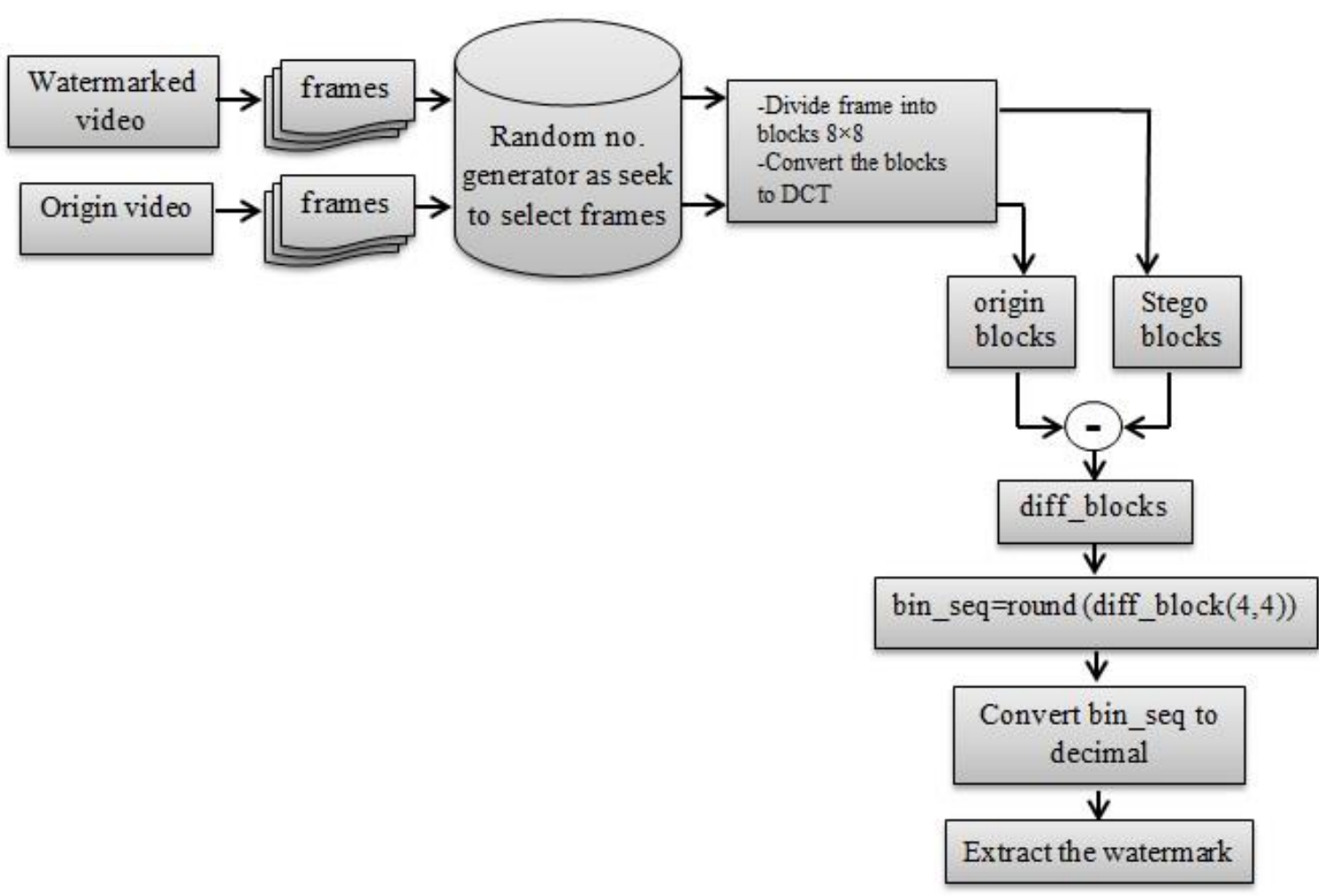

Figure(2) Watermark extraction algorithm based on DCT

\subsection{SVD based on Watermark embedding algorithm}

Step1:Read watermark image.

Step2:Convert RGB watermark to GRAY.

Step3:Resize the watermark to $40 \times 40$.

Step4:Generate random numbers within the range 1-no. of rows in watermark.

Step5:Read video.

Step6:Generate random numbers within the range 1-no. of frames in video.

Step7:Convert the values of watermark rows to binary sequence (bin_seq).

Step8:Embedding binary sequence into random frames using the following steps:

- Divide frame into blocks $8 \times 8$.

- Convert the blocks to SVD, from the diagonal values of V Take the minimum value.

- If (bin_seq(i)=1).

- then min_val $=$ min_val +10 .

- else no action.

- end.

- apply ISVD to blocks.

Step9: Rewrite the video 


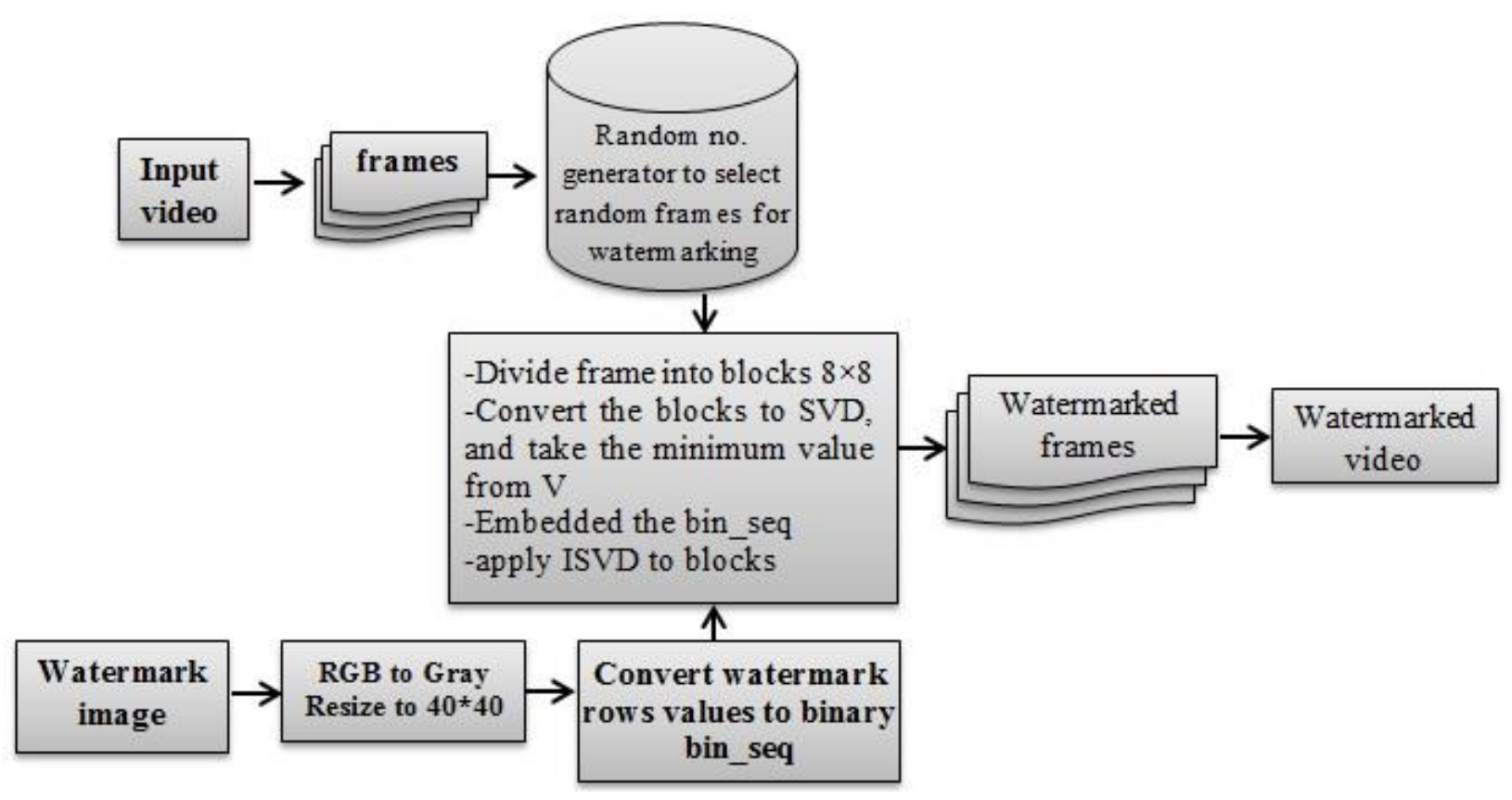

Figure(3) illustrates Watermark embedding algorithm based on SVD

\subsection{SVD based on Watermark Extraction algorithm}

Step1:Read the origin video(video1) and the watermarked video(video2).

Step2:Generate the same random numbers within the range 1-no. of frames depending on seek, and use them to take the frames from (video1) and (video2) .

Step3: Divide frames of video1\& video2 into blocks $8 \times 8$.

Step4:Convert the blocks of video1 to $\mathrm{SVD} \&$ the blocks of video2 to $\mathrm{S}_{1} \mathrm{~V}_{1} \mathrm{D}_{1}$.

Step5:Take the diagonal values from $\mathrm{V} \& \mathrm{~V}_{1}$, and calculate the differences between them .

- $\mathrm{VV}=\mathrm{V}_{1^{-}} \mathrm{V}$.

- $\mathrm{X}=\Sigma \mathrm{VV}$.

- If $(X>=10)$.

- then the embedded bit is 1 .

- else the embedded bit is 0 .

- end.

Step6:Convert the extracted bits to decimal values to create the rows of watermark. Step7:Rearrenge the rows to build the original watermark based on the 40 random numbers generated within the range 1-no. of rows using the order seek. 


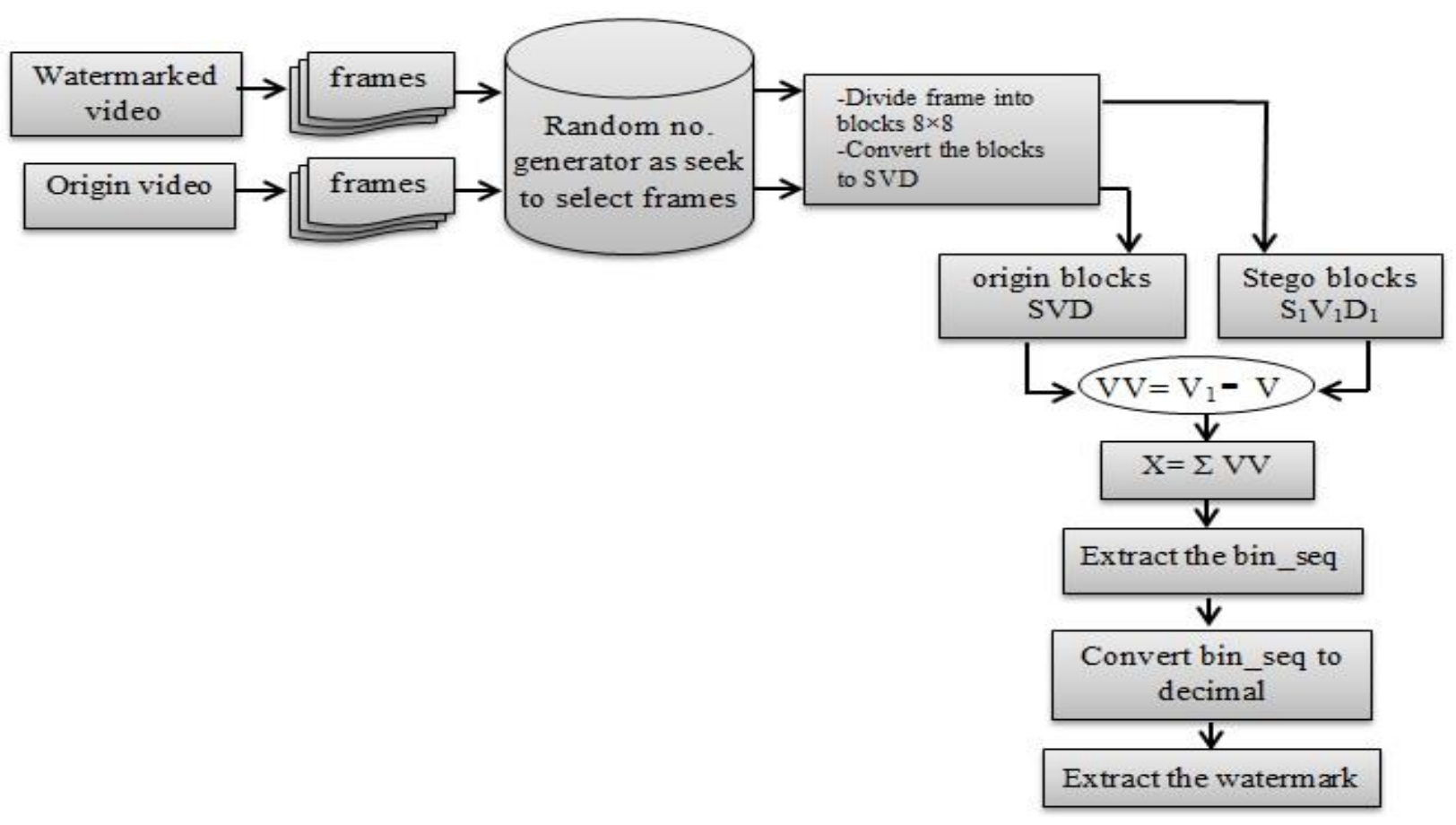

Figure(4) Watermark extraction algorithm based on SVD

\section{Experimental Results and Comparative Analysis}

The performance of the proposed watermarking method is evaluated using three standard measurements: Mean squared error (MSE) , Signal to Noise Ratio (PSNR) for measuring imperceptibility of the both methods MSE, PSNR and NC can be obtained using the following equation[12].

$$
\text { MSE }=\frac{1}{m * n} \sum_{i=0}^{m-1} \sum_{j=0}^{n-1}[I(i, j)-K(i, j)]^{2}
$$

where I and $\mathrm{K}$ represent the original and watermarked frames of video , $\mathrm{m}$ and $\mathrm{n}$ are the number of rows and columns in the input images[13] .

$P S N R=10 * \log _{10}\left(\frac{255^{2}}{M S E}\right)$

and the Normalized Cross Correlation (NC) is a metric commonly used to evaluate the degree of similarity between two images or frames[13].

It measures the correlation between the original and the extracted watermark frames before the being embedded and the frames after being the embedded. It is also used to measure the approximation of watermark before landfill and watermark after extracting from video. The NC value is confused if it is equal to or greater than 0.75 and $\mathrm{NC}$ is calculated using the following equation[14].

$N C=\frac{\sum_{i=0}^{N=1} w(i) \times w^{*}(i)}{\sum_{i=0}^{N-1}(w(i))^{2}}$ 
Where $\mathrm{w}(\mathrm{i})$ and $w^{*}(i)$ are the original watermark text and the extracted watermark text[9].

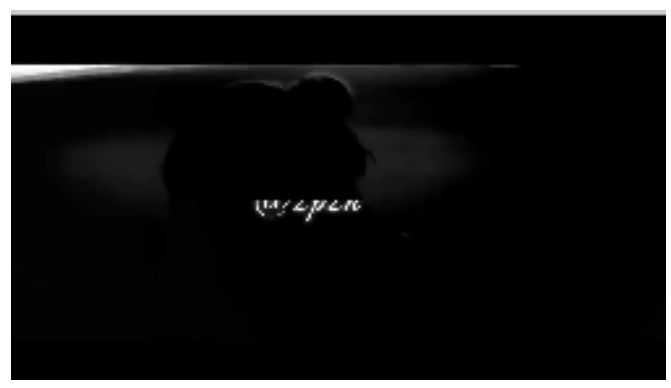

a-Original frame

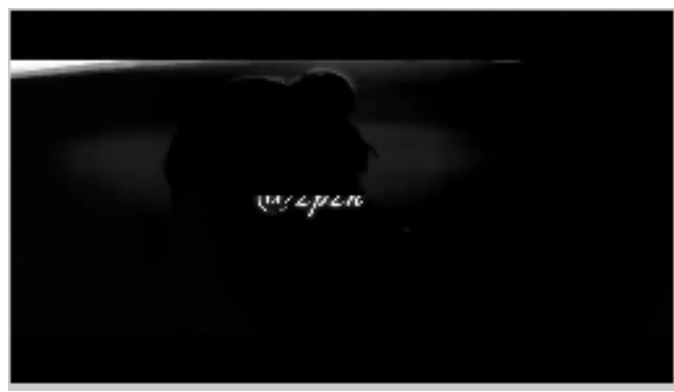

b- watermarked frame

Figure (5) shows original frame and watermarked frame using SVD

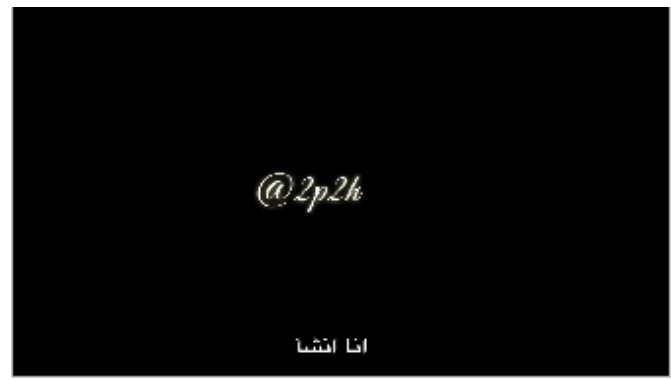

a-Original frame

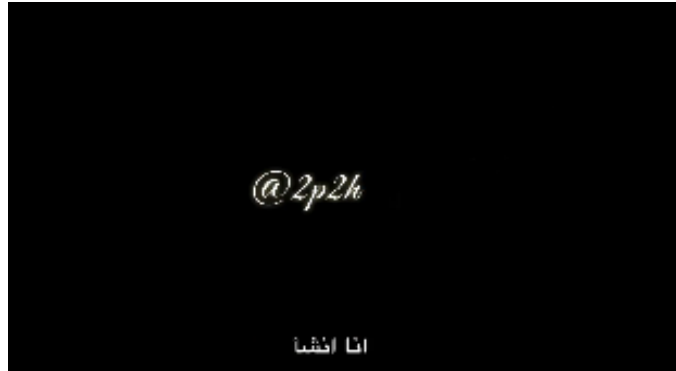

b- watermarked frame

Figure (6) shows original frame and watermarked frame using DCT

\section{Results \& discussion}

In this study, we used Matlab ver.2013a language to implement the proposed video watermarked methods. We also used AVI video of 890 frames, each frame of size $1280 \times 720$.

Table(1) show the comparison results of the original frame before and after the embedded of the watermark in it. Note from Table 1 that the PSNR value is 29.440 for DCT before and after embedded, which is better than the PSNR value for SVD and for the MSE values, the results were better for DCT than SVD. This indicates that the DCT method is more imperceptible and the Value of NC in both methods was equal to 1 This indicates the robustness of both methods. 
Table(1): quality metrics of watermark video

\begin{tabular}{|c|c|c|c|}
\hline Method & $\begin{array}{c}\text { Average } \\
\text { PSNR }\end{array}$ & $\begin{array}{c}\text { Average } \\
\text { MSE }\end{array}$ & $\begin{array}{c}\text { Average } \\
\text { NC }\end{array}$ \\
\hline DCT & 29.4401 & 0.0035 & 1 \\
\hline SVD & 22.74 & 0.0175 & 1 \\
\hline
\end{tabular}

Table (2) show the value of PSNR, MSE and NC Obtained when comparing the watermark before the embedded with the watermark after extracting it from the frame of video where the results proved that the watermark was not affected when using the embedded methods.

Table(2):Original watermark vs. extracted watermark

\begin{tabular}{|c|c|c|c|}
\hline Method & PSNR & MSE & NC \\
\hline DCT & infinity & 0 & 1 \\
\hline SVD & infinity & 0 & 1 \\
\hline
\end{tabular}

The performance method of DCT and SVD method was evaluate by performing several different signal processing attack on the watermark before embedding in the video, the video was subjected to the same attack after embedding the watermark. Performance was evaluated through several metrics.

The first metric is PSNR value decide the quality measurement between the original watermark and the watermark retrieved from video. the higher value of the PSNR means the best quality of the watermark included in the video and retrieved from the same vide the value of NC mean the degree of similarity between the original watermark and the recovered from video, the value of NC gave one or very closed to one, these values indicate a large similarity between the original watermark and recovered watermark. The two method DCT and SVD demonstrate that the highly robust against the different attacks which had been attack before and after embedded the watermark in video as shown in table(3). 
Table(3): different noise attacks with resulting quality metrics for extracted watermark

\begin{tabular}{|c|c|c|c|c|c|c|}
\hline Attack & \multicolumn{3}{|c|}{ DCT } & \multicolumn{3}{|c|}{ SVD } \\
\hline \multirow[t]{3}{*}{ Without noise } & & & & & & \\
\hline & PSNR & MSE & NC & PSNR & MSE & NC \\
\hline & infinity & 0 & 1 & infinity & 0 & 1 \\
\hline \multirow[t]{3}{*}{ Salt \& pepper } & & & 7 & & & \\
\hline & PSNR & MSE & NC & PSNR & MSE & NC \\
\hline & 22.93 & 330 & 0.955 & 30.58 & 56.87 & 0.992 \\
\hline \multirow[t]{3}{*}{ Gaussian } & & & & & & \\
\hline & PSNR & MSE & NC & PSNR & MSE & NC \\
\hline & 24.80 & 215 & 0.969 & 14 & 2120 & 0.7 \\
\hline \multirow[t]{3}{*}{ Speckle } & & & & & & \\
\hline & PSNR & MSE & NC & PSNR & MSE & NC \\
\hline & Infinity & 0 & 1 & infinity & 0 & 1 \\
\hline \multirow[t]{3}{*}{ Poisson } & & & 7 & & & \\
\hline & PSNR & MSE & NC & PSNR & MSE & NC \\
\hline & 30.4 & 59.13 & 0.991 & 23.5 & 289 & 0.95 \\
\hline
\end{tabular}




\section{Conclusion}

This paper demonstrate that digital video watermarking is applyed using DCT and SVD transformations. By showing to the results of the experiments obtained, which were evaluated using of NC, MSE and PSNR, as well as compared with previous works, it easy to conclude that Our approach gives encouraging results versus various types of attacks .Consequently, the proposed system demonstrated it is useful and appropriate for both copyright protection and content authentication applications.

\section{References}

[1] Gunjan M., Tarun K. and Gaurav A.,29-36.(5)(2017).

[2] Yogendra S., Jitender K. and Yogesh R.,2491-2497.(11) (2015).

[3] Rosemary K. and Mausumi G, 08, August-(2015).

[4] Khalil I. AlSaif and Meaad M. Salih .,Vol.12 No.4(2013).

[5] Khalil I. AlSaif and Meaad M. Salih, Vol.12,No.1(2013).

[6]Antonio.C and Mark.N,Digital Color image ownership Authentication Efficient and Robust watermarking ia a Hyberid Domain" San Francisco Culhuacan Coyoacan,vol.26,NO.2,Jun(2017).

[7] Dhanashree S. Shedge ," A Secure and Reliable Video Watermarking based on Different Wavelet Transform, Vol. 5, Issue 8, August(2017).

[8]Tahani A., Ali A., Lama R. and Hiba M., “A Robust Video Watermarking Algorithm”, Journal of Computer Science, vol. 4, no. 11, pp:910-915.(8)(2008).

[9] Jitender Kumar and Yogesh Rathi, Vol. 6 (3), 2015, 2491-2497,R2(2015).

[10]Chitrasen and Tanuja K., Vol. 7, Issue 10, ( Part -1) October 2017, pp.87-92.(2)(2017).

[11] U.Shyamala Devi1, S.Poongodi2 and Dr.B.Kalavathi , Vol-3, Issue-3, 2017,R4.(10)(2017). 
[12]Bedi S.S., Kumar A., and Kapoor P.," 46.1:46.7.(1)(2009).

[13] S.maruthuperumal and Dr.V.Vijaya Kumar 15-No.3.(7)(2011).

[14]Dhanashree S and Shedg, ISO 3297:2007. 